ARTICLE

\title{
Neutralizing activity of Sputnik $V$ vaccine sera against SARS-CoV-2 variants
}

Satoshi Ikegame, Mohammed N. A. Siddiquey ${ }^{2,4}$, Chuan-Tien Hung (1) 1, Griffin Haas', Luca Brambilla (1) 1, Kasopefoluwa Y. Oguntuyo ${ }^{1}$, Shreyas Kowdle (1) ${ }^{1}$, Hsin-Ping Chiu' ${ }^{1}$ Christian S. Stevens ${ }^{1}$, Ariel Esteban Vilardo ${ }^{3}$,

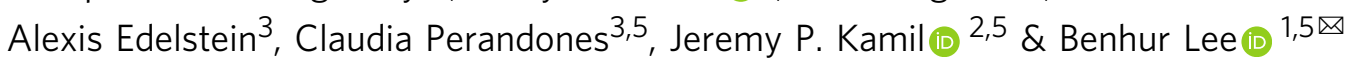

Severe acute respiratory syndrome coronavirus 2 (SARS-CoV-2) has infected at least 180 million people since its identification as the cause of the current COVID-19 pandemic. The rapid pace of vaccine development has resulted in multiple vaccines already in use worldwide. The contemporaneous emergence of SARS-CoV-2 'variants of concern' (VOC) across diverse geographic locales underscores the need to monitor the efficacy of vaccines being administered globally. All WHO designated VOC carry spike (S) polymorphisms thought to enable escape from neutralizing antibodies. Here, we characterize the neutralizing activity of postSputnik $\vee$ vaccination sera against the ensemble of $S$ mutations present in alpha (B.1.1.7) and beta (B.1.351) VOC. Using de novo generated replication-competent vesicular stomatitis virus expressing various SARS-CoV-2-S in place of VSV-G (rcVSV-CoV2-S), coupled with a clonal 293T-ACE2 + TMPRSS2 + cell line optimized for highly efficient S-mediated infection, we determine that only 1 out of 12 post-vaccination serum samples shows effective neutralization $\left(\mathrm{IC}_{90}\right)$ of rcVSV-CoV2-S: B.1.351 at full serum strength. The same set of sera efficiently neutralize $S$ from B.1.1.7 and exhibit only moderately reduced activity against $S$ carrying the E484K substitution alone. Taken together, our data suggest that control of some emergent SARS-CoV-2 variants may benefit from updated vaccines.

\footnotetext{
${ }^{1}$ Department of Microbiology at the Icahn School of Medicine at Mount Sinai, New York, NY, USA. ${ }^{2}$ Department of Microbiology and Immunology, Louisiana State University Health Shreveport, Shreveport, LA, USA. ${ }^{3}$ National Administration of Laboratories and Health Institutes of Argentina (ANLIS) Dr. Carlos G. Malbrán, Buenos Aires, Argentina. ${ }^{4}$ These authors contributed equally: Mohammed N. A. Siddiquey, Chuan-Tien Hung. ${ }^{5}$ These authors jointly supervised this

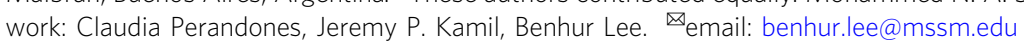


$\mathrm{n}$ the 15 months since its emergence in late $2019^{1}$, SARS-CoV2 has caused over 131 million confirmed COVID-19 cases worldwide, leading to at least 2.85 million deaths ${ }^{2}$. SARSCoV-2 is closely related to two other zoonotic betacoronaviruses, MERS-CoV and SARS-CoV, that also cause life-threatening respiratory infections ${ }^{3}$.

This global health emergency has spurred the development of COVID-19 preventive vaccines at an unprecedented pace. Six are already authorized for human use across the globe ${ }^{4-9}$. These vaccines focus on the SARS-CoV-2 spike protein (S), due to its critical roles in cell entry. Indeed, the presence of serum neutralizing antibodies directed at S correlate strongly with protection against COVID-1910,11. Although these six vaccines are efficacious, the recent emergence of SARS-CoV-2 variants has reignited concerns that the pandemic may not be so easily brought under control.

In December 2020, the United Kingdom reported the sudden emergence of a SARS-CoV-2 lineage, termed B.1.1.7 (501Y.V1, VOC 202012/01), which was designated as the first SARS-CoV-2 variant of concern (VOC). The lineage had rapidly increased in prevalence since first being detected in November $2020^{12}$. Its genome showed an unusually high number of non-synonymous substitutions and deletions, including eight in the S gene, suggesting a substantial degree of host adaptation that may have occurred during prolonged infection of an immunocompromised person $^{13}$. The B.1.1.7 lineage has now been shown to exhibit enhanced transmissibility ${ }^{14}$ as well as an increased case fatality rate $^{15,16}$

Soon afterwards, two additional SARS-CoV-2 VOC, B.1.351 and P.1, were reported from S. Africa and Brazil, respectively, which each showed substantial escape from neutralizing antibodies elicited by first wave pandemic viruses, leading to documented cases of re-infection ${ }^{17-19}$. The $S$ genes of B.1.351 and P.1 viruses each carry a number of mutations, but include three in the receptor binding domain $(\mathrm{RBD})$ that are particularly notable, the S: N501Y substitution, found in B.1.1.7, alongside polymorphisms at positions 417 and $484, \mathrm{~K} 417 \mathrm{~N} / \mathrm{T}$ and E484K. S: E484K had already been identified in multiple independent laboratories to confer escape from convalescent sera and monoclonal antibodies $^{20-22}$. As expected, the P.1 and B.1.351 variants escape or resist neutralization by first wave convalescent sera, as well as antibodies elicited by COVID-19 vaccines ${ }^{23-27}$.

Although the P.1 and B.1.351 lineages are dominant in Brazil and S. Africa, unlike B.1.1.7 they have not increased greatly in number in the United States since originally being detected here. In contrast, the E484K polymorphism is recurrently emergent, and is found in a number of other lineages that are increasing in the U.S. and other countries. For example, a B.1.526 sub-lineage carrying E484K in recent weeks has expanded more rapidly than B.1.1.7 28,29 , which may be indicative of the ability of S: E484K variants to penetrate herd immunity. The P.2 lineage, originally detected in Rio de Janeiro, carries only the E484K mutation in the RBD and has spread to other parts of South America, including Argentina $^{30}$.

The six COVID-19 vaccines currently in use around the world employ different strategies, and do not all incorporate the two proline substitutions that "lock" S into the pre-fusion conformer. Vaccines that do not utilize pre-fusion "locked" S are expected to produce lower levels of neutralizing antibodies, and hence may be less efficacious against infection, even if they do protect against severe COVID-1931,32. Indeed, a two-dose regimen of the AstraZeneca ChAdOx1 based vaccine, which does not use a "locked" S, did not protect against mild-to-moderate COVID-19 in S. Africa, where 93\% of COVID-19 cases in trial participants were caused by the B.1.351 variant ${ }^{33}$. Like the AstraZeneca ChAdOxl vaccine, the Sputnik V vaccine (Gam-COVID-Vac) is based on adenovirus vectored expression of a native $S$ sequence, rather than a pre-fusion "locked" $\mathrm{S}^{34}$. Although the Sputnik V vaccine has a reported vaccine efficacy of $91.6 \%$ in the interim analysis of Phase 3 trials held in Russia between September 7 and November 24, 2020, none of the VOC mentioned above nor independent lineages containing the $\mathrm{E} 484 \mathrm{~K}$ mutation were prevalent in Russia during this time period. Since the Sputnik vaccine is now in use not only in Russia, but also in countries like Argentina, Mexico, and Hungary, where some of the VOC and emerging lineages bearing the $\mathrm{E} 484 \mathrm{~K}$ mutation are more widespread, it is critical to assess the neutralizing activity of Sputnik vaccine elicited antibody responses against these cognate VOC and mutant spikes.

This study characterizes the neutralization activity of sera from a dozen Sputnik V vaccine recipients in Argentina. Our work was spurred by Argentina's nascent genomic surveillance efforts, which detected multiple independent lineages with S: E484K (B.1.1.318 and P.2) and/or S: N501Y substitutions (B.1.1.7 and P.1) in common, just as Argentina had started rolling out its vaccination campaign, which commenced on December 29, 2020. Here, we generated isogenic replication-competent vesicular stomatitis virus bearing the prevailing wild-type $(\mathrm{WT}=\mathrm{D} 614 \mathrm{G})$ SARS-CoV-2 S (rcVSV-CoV2-S), or the B.1.1.7 (alpha variant), B.1.351 (beta variant) or E484K mutant $S$ and used them in a robust virus neutralization assay. Our results show that Sputnik V vaccine sera effectively neutralized S: WT and S: B.1.1.7. viruses, albeit with highly variable titers. The same sera, however, exhibited moderate and markedly reduced neutralization titers, respectively, against S: E484K and S: B.1.351. Analyses of dose response curves indicate that S: B.1.351 exhibits resistance to neutralizing sera in a manner that is qualitatively different from the E484K mutant. Taken together, our data argue that surveillance of the neutralizing activity elicited by vaccine sera will be necessary on an ongoing basis. Viral neutralization assays can indicate which SARS-CoV-2 variants are likely capable of transmission in the face of vaccine elicited immunity, and whether updated vaccines will be needed to control their emergence and spread.

\section{Results}

Replication-competent VSV expressing SARS-CoV-2 spike proteins. Several groups have now generated replicationcompetent VSV expressing SARS-CoV-2 spike in place of VSVG (rcVSV-CoV2-S $)^{35-38}$. These rcVSV-CoV2-S can be used in BSL-2 compatible virus neutralization assays (VNAs), which correlate very well with VNAs using live SARS-CoV-2 (Spearman's $r>0.9$ across multiple studies). rcVSV-CoV2-S has been assessed as a candidate vaccine $e^{37,39}$, and used in forward genetics experiments to generate antibody escape mutants or perform comprehensive epitope mapping studies ${ }^{20,38,40}$. Indeed, the now concerning E484K mutation, present in many variants of concern (VOC), was identified as an antibody escape mutation using rcVSV-CoV-2-S 20,38 .

However, many groups passage their rcVSV-CoV-2-S extensively in Vero cells after the initial rescue, either to generate higher titer stocks and/or to remove confounding components such as the vaccinia virus expressing T7polymerase and/or transfected VSV-G, both of which were deemed necessary for efficient rescue ${ }^{38}$. Serial passage of rcVSV-CoV-2-S in Vero cells invariably leads to mutations in the S1/S2 furin cleavage site, as well as truncations in the cytoplasmic tail of the $\mathrm{S}$ protein ${ }^{39}$. The latter promotes $\mathrm{S}$ incorporation into VSV without compromising the conformational integrity of the ectodomain, whereas the former is problematic when assessing the neutralization sensitivity and 
structure-function phenotype of Spike VOC with multiple mutations that likely have complex epistatic interactions.

To generate rcVSV-CoV2-S containing different variants or mutants on demand, without the need for extensive passaging, we developed a robust reverse genetics system and VNA which leverages the cell lines we previously developed for a standardized SARS-CoV-2 VNA that correlates well with live virus neutralization $^{41}$. Salient improvements include the addition of a hammerhead ribozyme immediately upstream of the 3 ' leader sequence, which cleaves in cis to give the exact 3 ' termini, the use

a

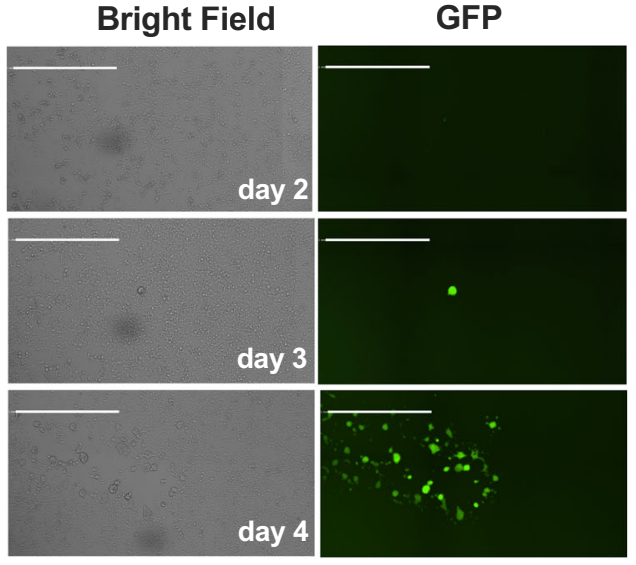

C

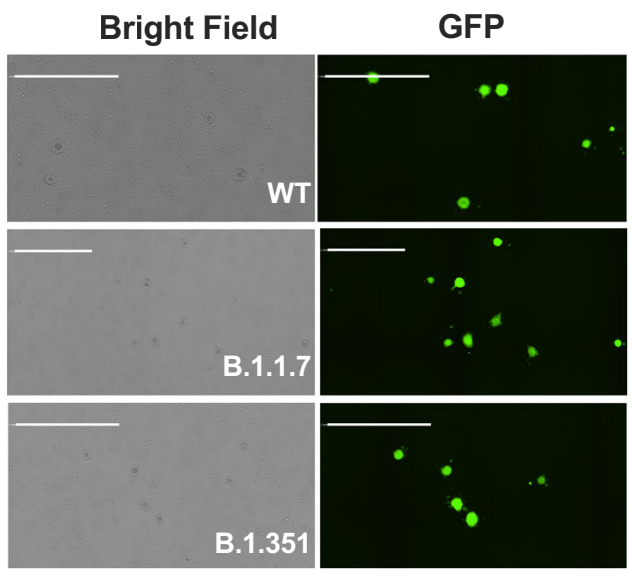

of a codon-optimized T7-polymerase which alleviates the use of vaccinia-driven T7-polymerase, and a highly permissive and transfectable 293T-ACE2 + TMPRSS2 clone (F8-2) ${ }^{41}$ (Supplementary Fig. 1). A 6-plasmid transfection into F8-2 cells resulted in GFP + cells 2-3 days post-transfection (dpt), which turned into foci of syncytia by $4-5 \mathrm{dpt}$ indicating virus replication and cell-to-cell spread (Fig. 1a). Transfer of F8-2 cell supernatant onto interferon-defective Vero-TMPRSS2 cells allowed for rapid expansion of low-passage viral stocks that maintain only the engineered Spike mutations. Clarified viral supernatants from

b

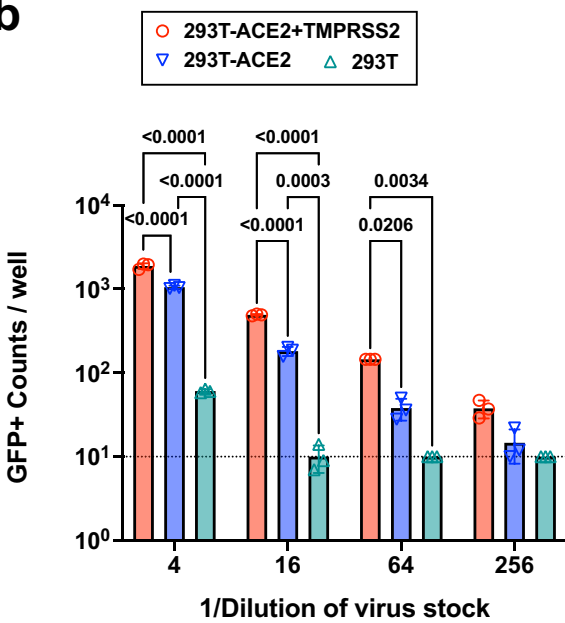

d

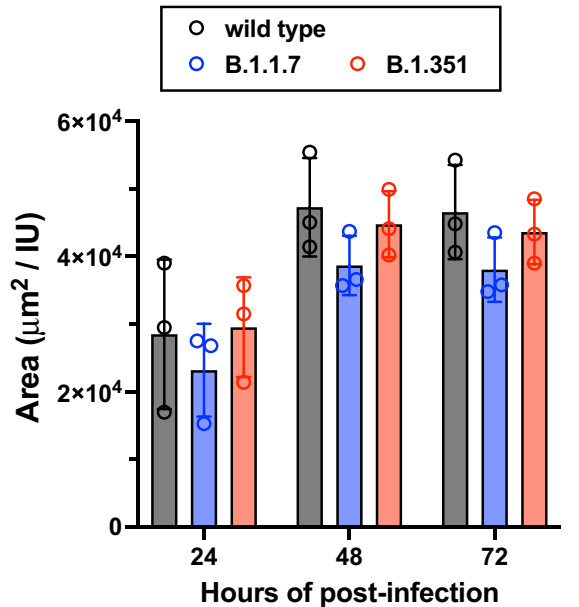

Fig. 1 Replication-competent VSV bearing wild-type and variant SARS-CoV-2 spike (rcVSV-CoV2-S). a De novo generation of rcVSV-CoV2-S, carrying an EGFP reporter, in transfected 293T-ACE2 + TMPRSS2 (F8-2) cells as described in Supplementary Fig. 1. Single GFP + cells detected at 2-3 days post-transfection (dpt) form foci of syncytia by $4 \mathrm{dpt}$. Images taken by Celigo imaging cytometer (Nexcelom) and are computational composites from the identical number of fields in each well. White bar equals 1 millimeter. Foci is representative of at least 5 independent rescues. $\mathbf{b}$ Entry efficiency of rcVSV-CoV2-S in parental 293T, 293T-ACE2, or 293T-ACE2 + TMPRSS2 cells. Serial dilutions of virus stocks amplified on Vero-TMPRSS2 cells were used to infect the indicated cell lines in 96-well plates in triplicates. GFP + cells were detected and counted by the Celigo imaging cytometer at $10 \mathrm{~h}$ post-infection (hpi). Symbols are individual data points from triplicate infections at the indicated dilutions. Bars represent the average of three replicates with error bars indicating standard deviation. Adjusted $p$-values from a two-way ANOVA with Tukey's multiple comparisons test are indicated in the graph. c rcVSV-CoV-2-S containing the prevailing WT (D614G) and VOC (B.1.1.7 and B.1.351) spikes were inoculated into one 6-well each of F8-2 cells (MOI 0.1) and subsequently overlaid with methylcellulose-DMEM to monitor syncytia formation. Representative images of syncytial plaques at $48 \mathrm{hpi}$ are shown (out of three replicates per virus per timepoint). White bar equals 1 millimeter. $\mathbf{d}$ shows the growth of GFP + area per infectious unit (IU) in the six-well plate. GFP + areas were imaged and measured by the Celigo imaging cytometer at 24,48 , and 72 hpi. IU was checked at $10 \mathrm{hpi}$ in the same well. GFP + area $\left(\mu \mathrm{m}^{2}\right)$ at the indicated timepoint was divided by the IU counted at $10 \mathrm{hpi}$ to normalize for input. Bar shows the average of triplicate experiments with error bar indicating standard deviation. Symbols represent individual data points. No statistically significant differences were detected between WT and VOC spikes in the size of GFP + syncytia at any given time point (two-way ANOVA as above, "ns" not indicated in graph). Source data are provided as a Source Data file. 
a

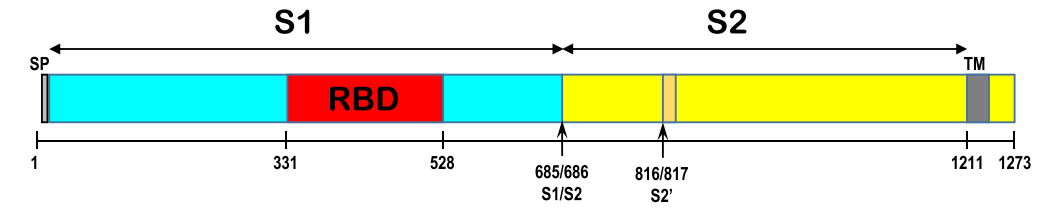

$\begin{array}{rccccccccccccccccccc} & 69 & 70 & 80 & 145 & 215 & 242 & 243 & 244 & 417 & 484 & 501 & 570 & 614 & 681 & 701 & 716 & 982 & 1118 & 1176 \\ \text { Ancestral } & \text { Y } & \text { Y } & \text { D } & \text { Y } & \text { D } & \text { L } & \text { A } & \text { L } & \text { K } & \text { E } & \text { N } & \text { A } & \text { D } & \text { P } & \text { A } & \text { T } & \text { S } & \text { D } & \text { V } \\ \text { Prevailing WT } & * & * & * & * & * & * & * & * & * & * & * & * & \text { G } & * & * & * & * & * & * \\ \text { B.1.1.7 } & - & - & * & - & * & * & * & * & * & * & \mathrm{Y} & \text { D } & \text { G } & \text { H } & * & \text { I } & \text { A } & \text { H } & * \\ \text { B.1.351 } & * & * & \text { A } & * & \text { G } & - & - & - & \text { N } & \text { K } & \text { Y } & * & \text { G } & * & \text { V } & * & * & * & * \\ \text { P.2 } & * & * & * & * & * & * & * & * & * & \text { K } & * & * & \text { G } & * & * & * & * & * & \text { F }\end{array}$

b

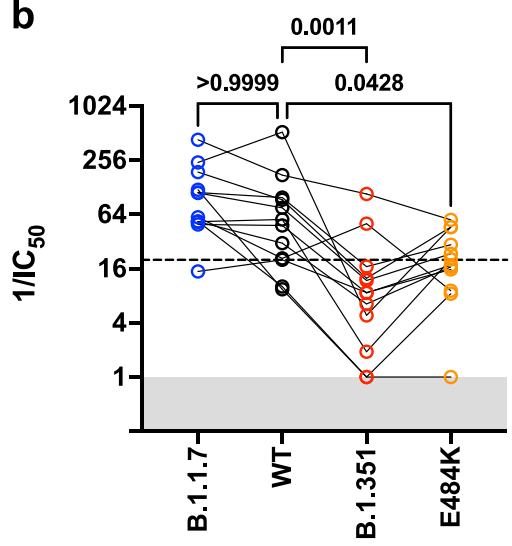

\begin{tabular}{|rcccc|}
\hline GMT & 87.1 & 49.4 & 7.9 & 17.0 \\
Lower 95\% Cl & 49.5 & 23.4 & 3.2 & 8.6 \\
Upper 95\% Cl & 154 & 105 & 19.5 & 33.8
\end{tabular}

C

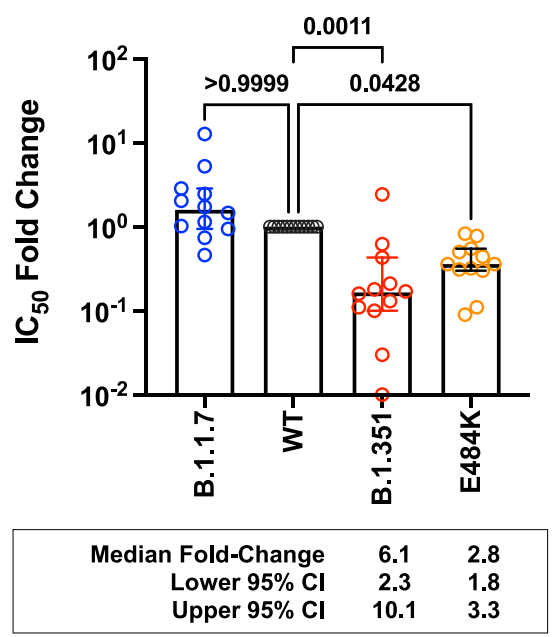

Fig. 2 Neutralization activity of antibody responses elicited by the Sputnik $\mathbf{V}$ vaccine. a Schematic of the Spike substitutions that make up the variants being evaluated in this study. The amino acid positions and corresponding "Ancestral" sequence of the Wuhan isolate is shown. The prevailing WT sequence now has a D614G substitution. All the variants and mutants have D614G. (b) Neutralization activity of individual serum samples against rcVSVCoV2-S with the WT (black circles), B.1.1.7 (blue circles), B.1.351 (red circles), or E484K (orange circles) spike proteins. Neutralization is represented by the reciprocal $50 \%$ inhibitory dilution factor $\left(1 / I_{50}\right)$. Sera samples with no appreciable neutralization against a given virus were assigned a defined $1 / I_{50}$ value of 1.0, as values $\leq 1$ are not physiological (Gray shaded area). Dashed line indicates the lowest serum dilution tested $(1 / I C 50=20)$. Geometric mean titers (GMT) and $95 \% \mathrm{Cl}$ for the neutralizing activity of all vaccine sera are indicated below each of the viral spike proteins examined. Adjusted $p$-values are indicated (non-parametric Friedman test with Dunn's multiple comparisons test). c For each serum sample, the fold-change in IC $\mathrm{C}_{50}$ (reciprocal inhibitory dilution factor) against the indicated variant and mutant spike proteins relative to its $I C_{50}$ against wild-type (WT) spike (set at 1$)$ is plotted. Adjusted $p$ values were calculated as in $\mathbf{b}$. Medians are represented by the bars and whiskers demarcate the $95 \% \mathrm{Cl}$. Neutralization dose-response curves were performed in triplicates, and the mean values from each triplicate experiment are shown as the single data points for each sera sample. Source data are provided as a Source Data file.

Vero-TMPRSS2 cells were aliquoted, sequenced verified, then titered on F8-2 cells to determine the linear range of response (Fig. 1b).

Next, we generated isogenic rcVSV-CoV2-S expressing the B.1.1.7, B.1.351, or E484K S to evaluate the neutralizing activity of Sputnik V vaccine sera from Argentina. Viruses bearing the alpha and beta variant spikes form spreading syncytia at a rate that was not significantly different from their wild-type counterpart (Fig. 1c, d).

Neutralization activity of Sputnik vaccine sera. The relevant spike substitutions that make up these variants are indicated in Fig. 2a. The characteristics of the vaccine recipient cohort $(n=12)$ receiving the two-dose regimen of the Sputnik vaccine are given in Table 1. At one month post-completion of the twodose regimen, the Sputnik V vaccine generated respectable virus neutralizing titers (VNT) against rcVSV-CoV2-S bearing the WT (D614G) and B.1.1.7 spike proteins (Fig. 2b). The geometric mean titer (GMT) and 95\% CI for WT (1//C 50 GMT 49.4, 23.4$105)$ in our cohort of vaccine recipients was remarkably similar to that reported in the phase III Sputnik vaccine trial (GMT 44.5, 31.8-62.2 $)^{42,43}$. However, GMT against B.1.351 and E484K was reduced by a median 6.8 - and 2.8 -fold, respectively, compared to WT (Fig. 2c).

Variable responses to SARS-CoV-2 variants. Sputnik vaccine recipients appeared to generate qualitatively different neutralizing antibody responses against SARS-CoV-2 that could be segregated into three different groups (Fig. 3). Group (A) sera showed reasonable VNT against wild-type (WT) and B.1.1.7 (Fig. 3a, e). However, the Hill slope of their neutralization curves for B.1.351 were relatively shallow $(h<0.40)$, resulting in a low IC50s and maximal neutralization of $50-60 \%$ even when extrapolated to full serum strength (Figs. 3e and 4). In contrast, Group (B) sera neutralized E484K and B.351 with similar potencies to WT and B.1.1.7, especially at high serum concentrations (Fig. 3b, e). This group of sera reveals that qualitatively different neutralizing responses can be generated that effectively neutralize B.1.351. Group (C) sera generally exhibited effective neutralization of WT, B.1.1.7, and even E484K at high serum concentrations, but not 
Table 1 Cohort characteristics of Sputnik vaccine recipients from ANLIS MALBRÁN (Buenos Aires, República Argentina).

\begin{tabular}{|c|c|c|c|c|c|}
\hline SPOO2 & Late $\mathrm{Dec} / 2020$ & Mid Jan/2021 & $(+)$ & $M$ & $40-45$ \\
\hline SPOO4 & Late $\mathrm{Dec} / 2020$ & Mid Jan/2021 & $(+)$ & M & $50-55$ \\
\hline SPO05 & Late Dec/2020 & Mid Jan/2021 & $(+)$ & M & $35-40$ \\
\hline SP006 & Late $\mathrm{Dec} / 2020$ & Mid Jan/2021 & $(+)$ & $\mathrm{F}$ & $35-40$ \\
\hline SP009 & Late $\mathrm{Dec} / 2020$ & Early Feb/2021 & $(+)$ & $\mathrm{F}$ & $30-35$ \\
\hline SP010 & Late $\mathrm{Dec} / 2020$ & Mid Jan/2021 & $(+)$ & $M$ & $30-35$ \\
\hline SP011 & Late $\mathrm{Dec} / 2020$ & Mid Jan/2021 & $(+)$ & $M$ & $40-45$ \\
\hline \multirow[t]{3}{*}{ SP012 } & Late Dec/2020 & Mid Jan/2021 & $(+)$ & M & $25-30$ \\
\hline & & & & Median age & 39.5 \\
\hline & & & & Range & $25-56$ \\
\hline SP013 & N.A. & N.A. & $(-)$ & $\mathrm{F}$ & $45-50$ \\
\hline
\end{tabular}

B.1.351 (Fig. 3c, e). The decreased potency and shallow Hill Slope resulted in $<90 \%$ neutralization of B.1.351 even at full serum strength (see next section). One serum sample (SP012) exhibited little to no neutralizing activity against WT, E484K and B.1.351, yet it neutralized B.1.1.7 as well as Group A-C sera (Fig. 3d, e). That these three groups exhibit qualitatively distinct neutralization patterns is further highlighted by the Hill slopes of their neutralization curves (Fig. 3f). Group A sera not only have the lowest slopes against B.1.351, but as a group, they have slopes significantly $<1$ against the other viruses (median $/ \mathrm{IQR}=0.4965$ / $0.2880-1.186)$. Group B sera mostly have slope values around 1 (median/IQR $=0.8855 / 0.7865-1.065)$ while Group C sera have the highest overall slopes (median/IQR $=1.348 / 0.8395-1.820$ ) that are significantly $>1$ (Fig. $3 f$ ).

Resistance to neutralization correlates with shallow Hill slopes. The Hill Slope of the neutralization curves against B.1.351 was significantly different from WT, B.1.1.7 and E484K (Fig. 4a). As a consequence, the maximal neutralization attainable when extrapolated to full serum strength was also significantly lower for B.1.351 compared to the rest (Fig. 4b). Conversely, the steep Hill slope for the E484K curves resulted in maximal neutralization potencies that were not significantly different from WT or B.1.1.7 despite significantly lower reciprocal $\mathrm{IC}_{50}$ values (compare Fig. $2 \mathrm{~b}$ with Fig. 4b). Notably, the maximal percent inhibition was strongly correlated with the Hill Slope for WT and VOC/mutant spikes across all valid pairs of sample values (Fig. 4 c, $n=45$ pairs), suggesting that antibody co-operativity likely plays a role at high serum concentrations (see "Discussion") 44 . While we acknowledge the limitations of extrapolating values from nonlinear regression curves, the striking correlation between slope and maximal percent inhibition attainable at full serum strength supports the robustness of our nonlinear regression model.

The heterogenous dose-response curves described in Figs. 3-4 is a property of Sputnik $\mathrm{V}$ vaccine elicited responses as soluble RBD-Fc inhibition of WT and VOC S-mediated entry produced classical dose response curves with Hill slopes close to -1.0 (Fig. 5). Both B.1.1.7 and B.1.351 were modestly but significantly more resistant to RBD-Fc inhibition (Fig. 5a, b). This is not surprising as both harbor the N501Y mutation known to enhance affinity of RBD for ACE2 $45-47$. However, this 1.5 to 2-fold increase in $\mathrm{RBD}-\mathrm{Fc} \mathrm{IC}_{50}$ for B.1.1.7 and B.1.351, respectively, does not explain the neutralization-resistant versus sensitive phenotype of B.1.351 versus B.1.1.7 in our virus neutralization assays. Furthermore, the E484K mutant was more sensitive to RBD-Fc inhibition than B.1.1.7 (Fig. 5c, d), and yet also remained more neutralization-resistant relative to B.1.1.7. Experimental measurements of both RBD and trimeric spike binding to ACE2 have revealed that the $\mathrm{E} 484 \mathrm{~K}$ mutation alone does not confer increase binding affinity for ACE2 unlike N501Y ${ }^{46,48}$. Our RBD-FC inhibition studies in the context of virus infection confirm and extend these results. Our data reinforces the notion that the mechanism underlying the increased neutralization resistance of E484K containing variants and mutants do not involve ACE2 binding affinity per se, but rather affects a key immunodominant epitope targeted by a significant class of human neutralizing antibodies, variably termed as RBM class II, RBS-B, or Cluster 2 antibodies $42,43,49,50$.

\section{Discussion}

A key public health concern related to emergent SARS-CoV-2 variants is that by incrementally accruing mutations that escape neutralizing antibodies, they will penetrate herd immunity and spread to reach unvaccinated individuals, some of whom will be susceptible to severe or fatal disease.

Three of the six COVID-19 vaccines currently in use worldwide, namely Moderna mRNA-1273, BioNTech BNT162b2, and Janssen Ad26.COV2.S, each express S harboring K986P and V987P substitutions (2P) within a loop abutting the central helix of the S2' membrane fusion machinery ${ }^{51-53}$. This modification locks the spike in a prefusion conformation and elicits higher titers of neutralizing antibodies ${ }^{54,55}$. The Janssen vaccine has an additional deletion in the furin cleavage site, while the yet-to-be approved Novavax vaccine contains arrays of stabilized spikes conjugated onto a nanoparticle (Table 2). Of the three vaccines that do not appear to make use of $2 \mathrm{P}$ Spike mutants, Gamaleya's Sputnik V and AstraZeneca's AZD1222 are adenovirus-vectored vaccines encoding native $S$. The third is CoronaVac, a preparation of inactivated SARS-CoV-2 virions. Although all six vaccines are highly efficacious at preventing severe COVID-19 outcomes, they do not all uniformly prevent infection. Moreover, in all cases thus far examined, these first generation vaccines are less effective against variants with certain non-synonymous substitutions in Spike, such as E484K. 
a

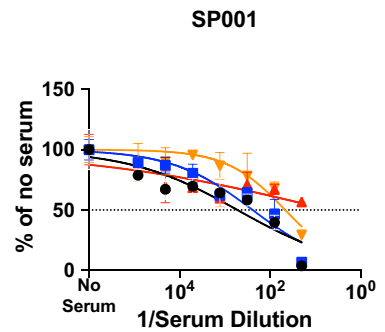

b

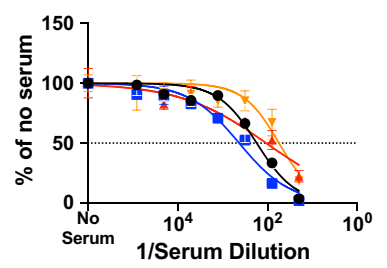

C

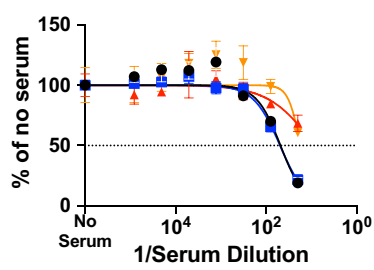

e
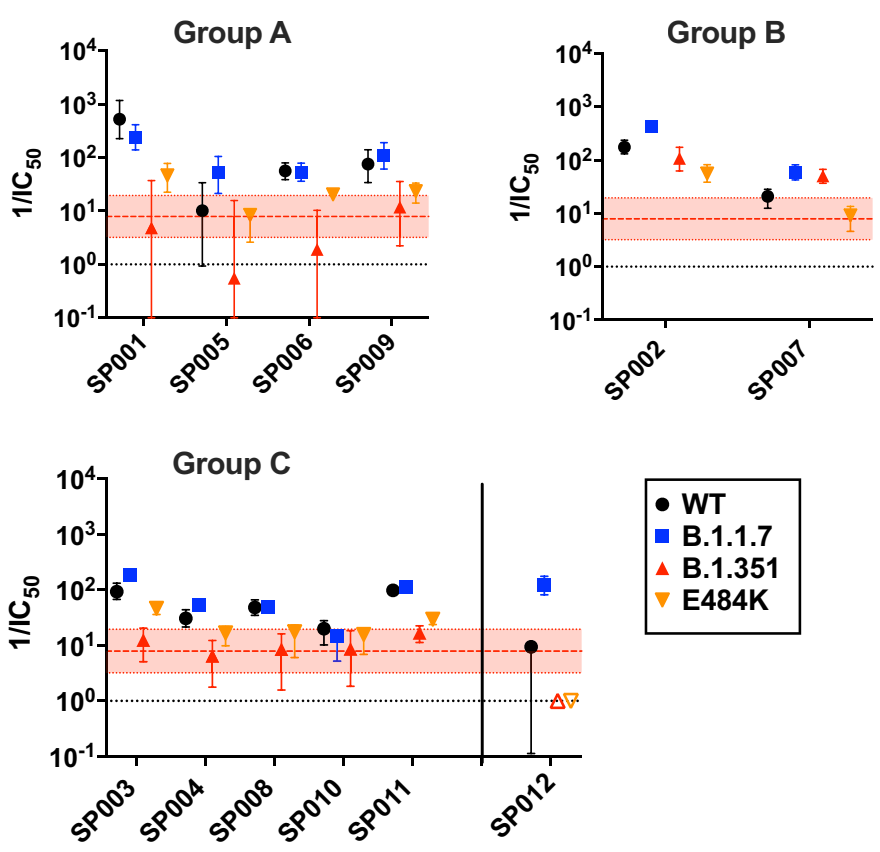

SP005

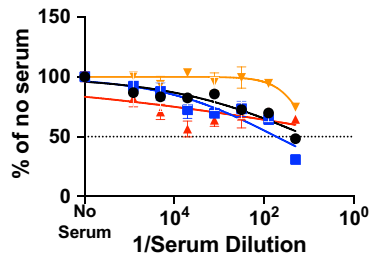

SP007

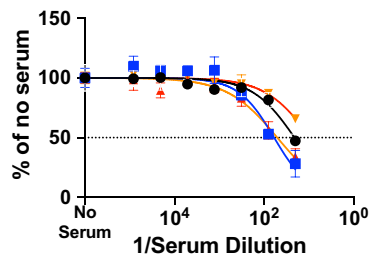

SP010

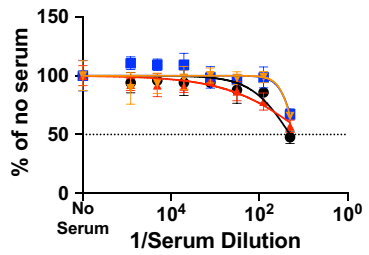

SP009

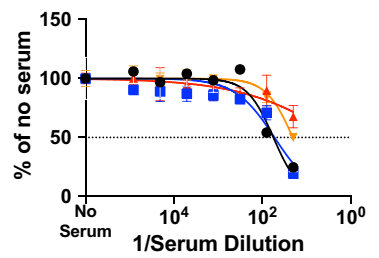

SP003

C

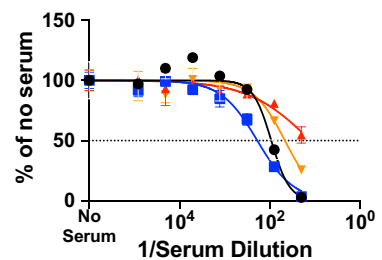

SP011

d

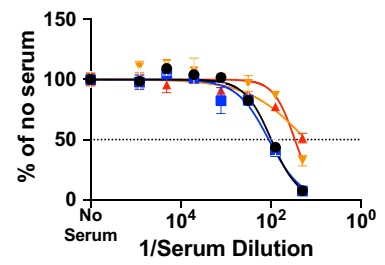

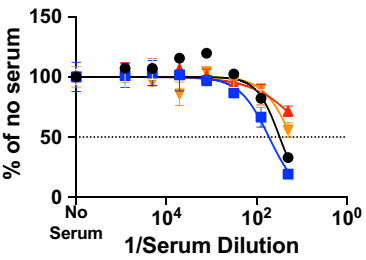

SP012

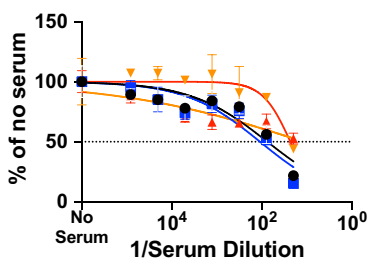

SP004

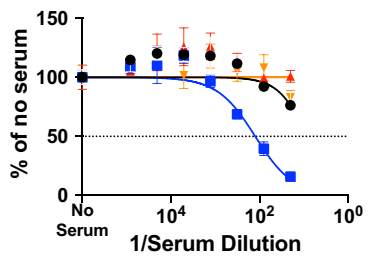

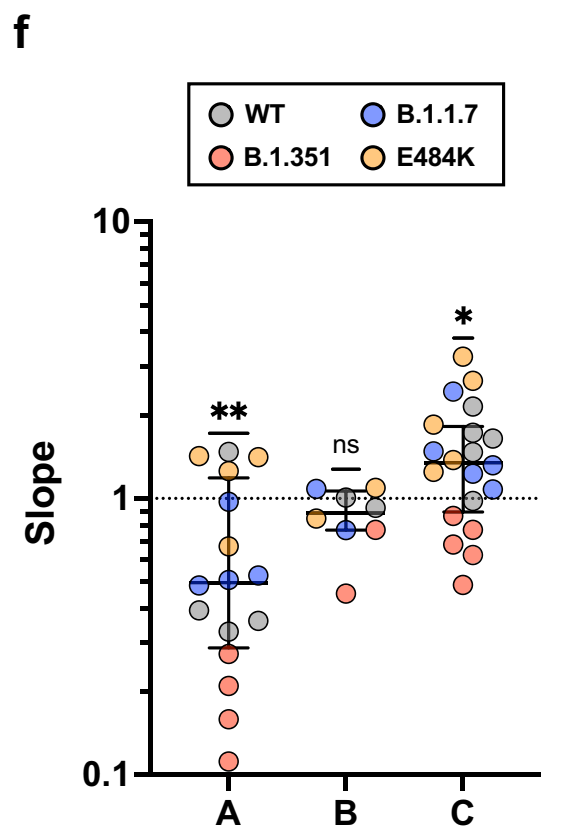

The most concerning variants are those with multiple mutations in the receptor binding domain (RBD) that confer both enhanced affinity for the hACE2 receptor and escape from neutralizing antibody responses ${ }^{17,24,27,33,56,57}$. B.1.351 and P.1 have in common three RBD substitutions (K417N/T, E484K and N501Y) whereas B.1.351, P.1 and B.1.1.7 contain the N501Y substitution. Although B.1.1.7 shows enhanced transmissibility and more severe disease outcomes ${ }^{52}$, it does not appear to be consistently more resistant to serum neutralizing responses elicited by vaccines or natural infection ${ }^{58,59}$. The same is not true, however, for the B.1.351 variant.

In live virus plaque reduction neutralization assays, sera from AstraZeneca vaccine recipients in South Africa exhibited 4.1 to 32.5 -fold reduction in neutralizing activity against B.1.351 ${ }^{33}$. The 
Fig. 3 Sputnik vaccine recipients generate qualitatively different neutralizing antibody responses against SARS-CoV-2. a-c Group A (SP001, SP005, SP006, SP012), Group B (SP002, SP007), and Group C (SP003, SP004, SP008, SP010, SP011) represent potentially distinct classes of virus neutralizing activity present in the sera samples analyzed. Full neutralization curves for all sera tested against all viruses bearing the variant and mutant spike proteins are shown. d shows a singular example of a serum that only neutralized the B.1.1.7 spike. Data shown at each serum dilution point are normalized mean+/- standard deviation from triplicate infections. e graphs the serum neutralizing titers (SNT $=1 / \mathrm{IC}_{50}$ ) and $95 \% \mathrm{Cl}$ extrapolated from the nonlinear regression curves shown in a-d $(n=12)$. Colored filled symbols represent the indicated viruses, open symbols in e represent assigned SNT values of 1.0 when no significant neutralization activity could be detected (SP012: B.1.351 and E484K). The dotted black line represents a reciprocal serum dilution of 1.0. The red dashed line and shaded boundaries represent the geometric mean titer and $95 \% \mathrm{Cl}$, respectively, for B.1.351 across all samples $(n=12)$. f The Hill slope values for all the neutralization curves are aggregated according to their groups. The different colored symbols in each group represent the indicated virus tested. The median (central bar) and interquartile range (whiskers) for each group are also shown. $p$-values (two-tailed) are from a non-parametric Wilcoxon-signed rank test comparing each group to a theoretical median of 1.0 (Group A, $n=16$, Group B, $n=8$, Group C, $n=20$ ). ${ }^{\star \star}=0.0065$, ${ }^{\star}=0.0110$, ns $=0.1953$. Source data are provided as a Source Data file.
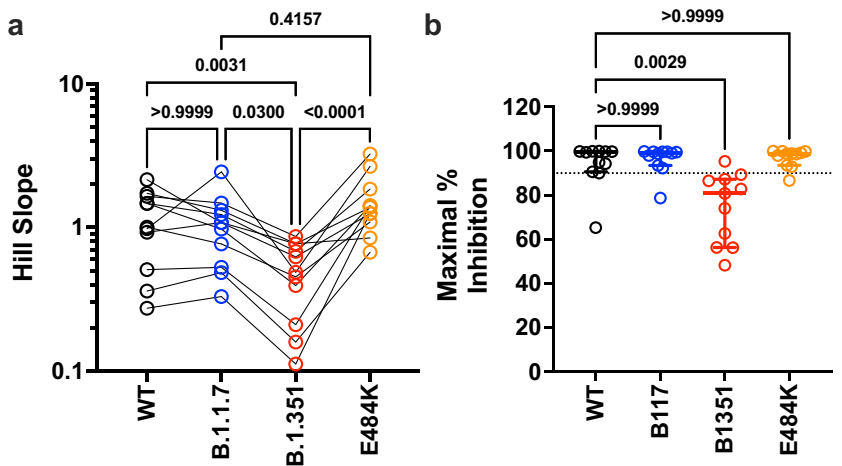

C

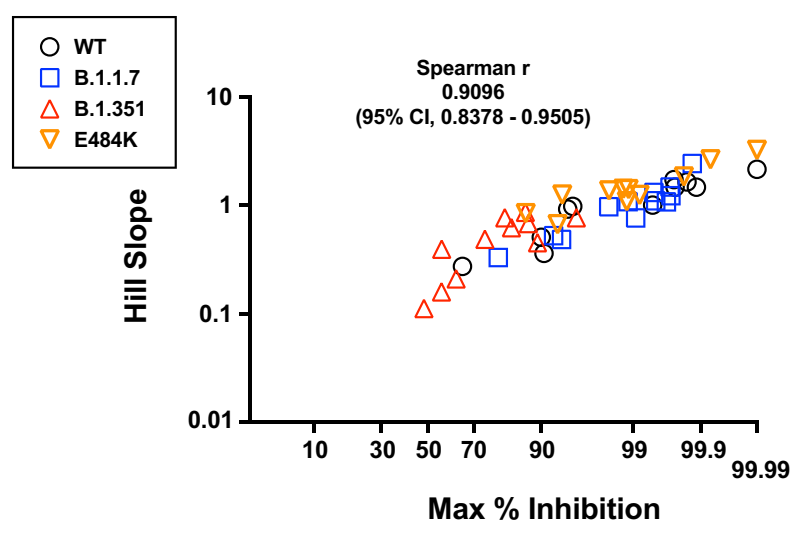

Fig. 4 Maximal inhibition and slope help to define the distinct classes of neutralizing sera in Sputnik vaccine recipients. a Paired comparison of Hillslopes from the neutralization curves of all samples except for SP012 where no significant neutralization was observed for viruses other than B.1.1.7. Adjusted $p$-values are indicated (non-parametric Friedman test with Dunn's multiple comparisons test, which assumes non-Gaussian distribution of values being analyzed). b Maximal percent inhibition (MPI) at full serum strength extrapolated from nonlinear regression of log(inhibitor) versus normalized response, variable slope curve. Model used is from PRISM v9.1.1 where $y=100 / 1+10^{(\log / C 50-x) \times \text { Hillslope }}$. Log IC50 and Hill slope values were obtained for each curve generated in Fig. 3. MPI $=100-Y$, when $X=0$ for reciprocal serum dilution of $1\left(10^{0}=1\right)$. Data points for one serum (SP012) against WT, B.1.351 and E484K could not be calculated because there was no best-fit value. The dotted line indicates $90 \%$ inhibition. Median (central bar) and interquartile values (whiskers) are indicated. Adjusted $p$-values was calculated as in a. Black, blue, red, and organe circles represent WT, B.1.1.7, B.1.351, and E484K viruses, respectively. c Correlation analysis of MPI versus the Hill Slope parameter for all sera samples tested against all spike proteins. SP012 was excluded for the abovementioned reasons. Non-parametric Spearman $r$-values and $95 \%$ confidence interval are shown. $x$-axis is plotted as an asymptotic cumulative probability scale as $x$ approaches 100\% (PRISM v9.1.1) only to resolve the many MPI values $>90 \%$. Source data are provided as a Source Data file. actual reduction is even more marked because 7 of 12 vaccine recipients who had neutralizing activity against the parental B.1.1 variant, had undetectable neutralization against the B.1.351 strain. Comparator sera from recipients of Moderna and BioNTech mRNA vaccines showed smaller, 6.5- to 8.6-fold reductions in neutralization ${ }^{60}$.

Here, we showed that sera from Sputnik vaccine recipients in Argentina had a median 6.1-fold and 2.8-fold reduction in GMT against B.1.351 and the E484K mutant spike, respectively. Even more revealing is their dose-response curves. When extrapolated to full serum strength, half of the sera samples failed to achieve an $\mathrm{IC}_{80}$ and only 1 out 12 achieved an $\mathrm{IC}_{90}$ against B.1.351 (Fig. 4a). Table 2 summarizes peer-reviewed studies that have tested postvaccination sera from the major vaccines against the $\mathrm{VOC} /$ mutant spikes used in this study. Our study shows a similar mean reduction in GMT (reciprocal IC50) against E484K and B.1.351 using 1-month post-Sputnik vaccine sera when compared to other vaccines. Our sample number is admittedly small but matches the median and modal number used in other studies to date. Nonetheless, we caution that comparing only the mean reduction in IC50 can be misleading as an aggregate measure of serum neutralizing activity. The neutralization curves for B.1.351 in our study are not classically sigmodal and have significantly shallower slopes than WT, B.1.17 and E484K, which result in $\leq$ $90 \%$ neutralization for all but one sample when extrapolated to full serum strength. The possible mechanisms for the varying slope values are discussed below.

E484K is present not only as part of an ensemble of RBD mutations present in B.1.351 and P.1, but in many of the 17 lineages detected from South America that carry it, such as P.2, E484K is the only RBD substitution (Supplementary Table 1). While the $\mathrm{E} 484 \mathrm{~K}$ substitution appears to be a common route of escape from many RBD-targeting monoclonal antibodies, it is somewhat surprising that a single mutation can confer a significant degree of neutralization resistance from polyclonal responses. Nonetheless, our data show that resistance conferred by $\mathrm{E} 484 \mathrm{~K}$ mutation be overcome by higher titer antibodies present in undiluted patient sera. But the neutralization resistance conferred by the suite of mutations present in B.1.351 appears qualitatively different. In the majority of cases, the slope of the dose response curve indicates a failure to neutralize even at full strength. We had previously shown that the doseresponse curve slope is a major predictor of therapeutic potency for HIV broadly neutralizing antibodies at clinically relevant concentrations ${ }^{44}$. Importantly, the slope parameter is independent of IC50 but is specifically related to an antibody's epitope class. Here, we show that defining the neutralization phenotype of a given spike variant or mutant by both its relative IC50 and slope provides a fuller characterization of serum neutralizing activity against SARS-CoV-2 and emergent VOC. 


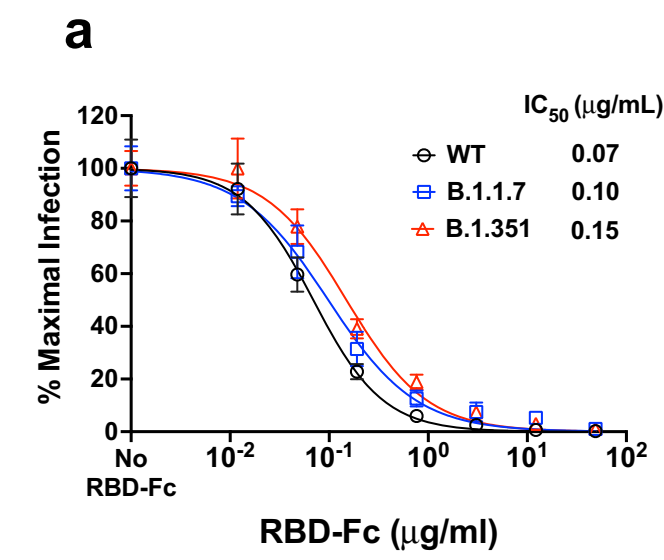

b
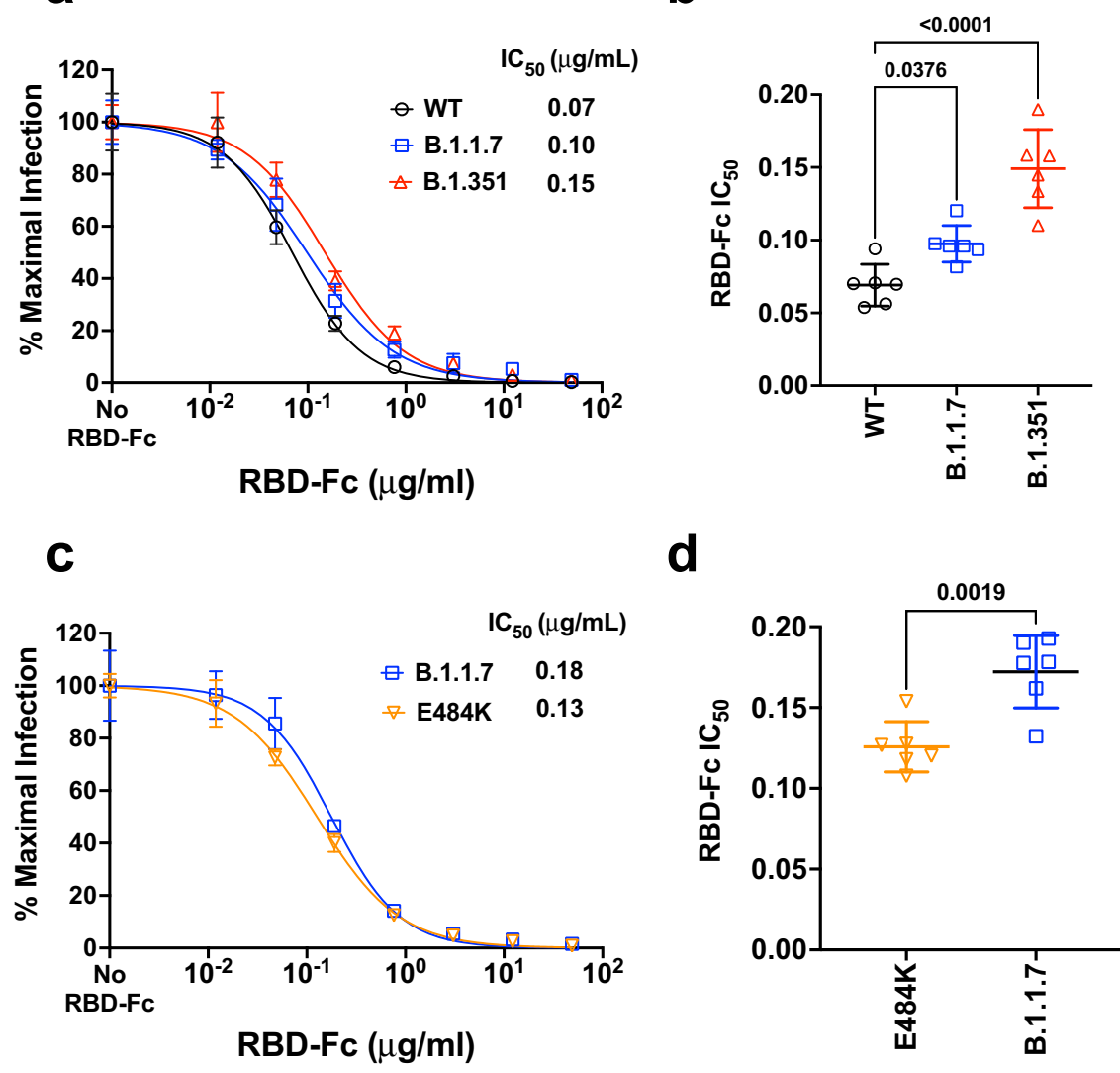

Fig. 5 Competitive inhibition of rcVSV-CoV2-S entry by soluble RBD-Fc. a Recombinant RBD-Fc was serially titrated with the infection inoculum containing a fixed amount of rcVSV-CoV2-S bearing WT or the indicated VOC spike proteins. $10 \mathrm{hpi}$, GFP+ cells were quantified by the Celigo image cytometer. Data points are means of six replicates with error bars representing standard deviation. The number of GFP+ cells in the absence of any RBD-Fc was set to $100 \%$ and used to normalize the infection response in the presence of increasing amounts of RBD-Fc. Log[inhibitor] versus normalized response variable slope nonlinear regression curves were generated using GraphPad PRISM (v9.1.0). b The IC50 values from each replicate dose-response curve generated for a given virus were grouped. The mean (central bar) and standard deviation (whiskers) for each group are indicated. Adjusted $p$-values are indicated (ordinary one-way ANOVA with Dunnett's multiple comparisons test are indicated). $\mathbf{c}$ is a repeat of the experiment done in $\mathbf{a}$ with the E484K mutant using a different preparation of recombinant RBD-Fc (see methods). B.1.1.7 serves as the common reference control. Data points are means of six replicates with error bars representing standard deviation. $\mathbf{d}$ The IC50 values were calculated as in $\mathbf{b}$. The mean (central bar) and standard deviation (whiskers) for each group are indicated. Two-tailed $p$-value (0.0019) from an unpaired $t$-test is shown. Source data are provided as a Source Data file.

The deletion of residue $242-244$ in the NTD of the B.1.351 spike appear to cause large-scale resurfacing of the NTD antigenic surface resulting in greater conformational heterogeneity ${ }^{46}$. Variable neutralization responses across such a heterogenous virus population may result in the shallow slopes $(<1)$ seen. Furthermore, three major classes of neutralizing antibodies (RBS-A, -B, and -C) identified from convalescent patients are sensitive to either the K417N (RBS-A) or E484K (RBS-B and -C) substitutions present in B.1.35161. On the other hand, at high serum concentrations, co-operative effects from low-affinity spike binding antibodies or increased spike occupancy by different classes of antibodies can result in the steep Hill slope observed. The steep Hill slopes (>1.0) observed for E484K suggest such cooperative effects might be occurring.

The emergence of variants is fluid situation. B.1.427/1.429, B.1.526, and B.1.617 are other emergent VOI/VOC that could be tested. These strains have substitutions in the RBD (L452R and $\mathrm{E} 484 \mathrm{~K} / \mathrm{Q}$ ) and elsewhere in the spike that might confer some degree of neutralization resistance in our in vitro assays. However, all vaccines are effective against most variants, and we do not know what degree of resistance in in vitro assays translate to a decrease in the real world efficacy of any given vaccine.
Although we stress that the Gameyla Sputnik V vaccine is likely to retain strong efficacy at preventing severe COVID-19, even in the case of infection by VOC, our data reveal a concerning potential of B.1.351, and to a lesser extent, any variant carrying the E484K substitution (e.g. P.2), to escape the neutralizing antibody responses that this immunization elicits. Furthermore, we acknowledge that in vivo protective efficacy can be derived from Fc effector functions of antibodies that bind but do not neutralize. In addition, an adenoviral vectored vaccine should induce potent cell-mediated immunity against multiple epitopes, which were not measured in our study. Nevertheless, given the crucial roles neutralizing antibodies play in preventing infection, our results suggest that updated SARS-CoV-2 vaccines may be necessary to eliminate the virus.

\section{Methods}

Cell lines. Vero-CCL81-TMPRSS2 (ATCC CCL-81 derived), HEK 293T-hACE2 (clone 5-7), and 293T-hACE2-TMPRSS2 (clone F8-2) cells (ATCC CRL-3216 derived) were described previously ${ }^{41}$, and were maintained in Dulbecco's modified Eagle's medium (DMEM, Gibco) containing 10\% fetal bovine serum (FBS, R\&D Systems). The HEK 293T-hACE2-TMPRSS2 cells were plated on collagen coated plates or dishes. BSR-T7/5 cells ${ }^{62}$ (RRID: CVCL_RW96), which stably express T7polymerase were maintained in DMEM with $10 \%$ FBS. 
Table 2 Summary of post-vaccine sera evaluated for neutralization potency against the indicated SARS-CoV-2 variants of concern (VOC).

Neutralization assay (IC 50 fold-reduction vs. WT)

\begin{tabular}{|c|c|c|c|c|c|c|c|}
\hline Vaccine & Company & $\begin{array}{l}\text { Spike } \\
\text { construct }\end{array}$ & $\begin{array}{l}\text { B.1.1.7 } \\
\text { (Alpha) }\end{array}$ & $\begin{array}{l}\text { P.1 } \\
\text { (Gamma) }\end{array}$ & B.1.351 (Beta) & $\begin{array}{l}\text { Number of } \\
\text { samples tested (n) }\end{array}$ & Reference (PMID) \\
\hline Ad26.COV2.S & Johnson\&Johnson & $2 \mathrm{P} \& \Delta$ Furin & $\leq 2 \times($ n.s. $)$ & NA & $\leq 5 \times$ & 8 & $33909009^{a}$ \\
\hline BNT162b2 & Pfizer/BioNTech & $2 \mathrm{P}$ & $2 \times$ & NA & $\leq 6.5 x$ & 10 & 33684923 \\
\hline BNT162b2 & Pfizer/BioNTech & $2 \mathrm{P}$ & $2 x$ (n.s.) & $6.7 \times$ & $35 x$ & 30 & 33743213 \\
\hline BNT162b2 & Pfizer/BioNTech & $2 \mathrm{P}$ & $3.3 x$ & NA & NA & 25 & 33743891 \\
\hline BNT162b2 & Pfizer/BioNTech & $2 \mathrm{P}$ & NA & NA & 7.9 & 25 & 33730597 \\
\hline mRNA-1273 & Moderna & $2 \mathrm{P}$ & $1.8 \times$ & NA & $\leq 8.6 \times$ & 12 & 33684923 \\
\hline mRNA-1273 & Moderna & $2 \mathrm{P}$ & (n.s.) & $4.5 \times$ & $28 x$ & 35 & 33684923 \\
\hline $\begin{array}{l}\text { BNT162b2 or } \\
\text { mRNA-1273 }\end{array}$ & $\begin{array}{l}\text { Pfizer/BioNTech or } \\
\text { Moderna }\end{array}$ & $2 \mathrm{P}$ & NA & $\leq 3 \times$ & NA & $15^{b}$ & 33567448 \\
\hline NVX-CoV2373 & Novavax & $3 Q-2 P$ & $2 \times$ & NA & NA & 28 & 33705729 \\
\hline AZD1222 & AstraZeneca & Native & NA & NA & $4 \times$ & 13 & 33725432 \\
\hline AZD1222 & AstraZeneca & Native & $8.9 x$ & NA & NA & 49 & 33798499 \\
\hline AZD1222 & AstraZeneca & Native & $2.1-2.5 x$ & NA & NA & 25 & 33743891 \\
\hline AZD1222 & AstraZeneca & Native & NA & NA & $9 x$ & 25 & 33730597 \\
\hline CoronaVac & Sinovac & Native & NA & NA & NA & N.D. & N.D. \\
\hline BBIBP-CorV & Sinopharm & Native & NA & $\begin{array}{l}\text { NA } \\
\text { E484K }\end{array}$ & $1.6 \times$ (n.s.) & 12 & 33870240 \\
\hline Sputnik V & Gamaleya & Native & (n.s.) & $\begin{array}{l}2.8 x \\
(E 484 K)\end{array}$ & $6.1 x$ & 12 & $33821288^{c}$ \\
\hline
\end{tabular}

VSV-eGFP-CoV2 spike ( $\Delta$ 21aa) genomic clone and helper plasmids. We cloned VSV-eGFP sequence into pEMC vector (pEMC-VSV-eGFP), which includes an optimized T7 promoter and hammerhead ribozyme just before the 5' end of the viral genome. The original VSV-eGFP sequence was from pVSV-eGFP, a generous gift of Dr. John Rose ${ }^{63}$.

We generated pEMC-VSV-eGFP-CoV2-S (Genbank Accession: MW816496) as follows: the VSV-G open reading frame of pEMC-VSV-eGFP was replaced with the SARS-CoV-2 S, truncated to lack the final 21 amino acids ${ }^{64}$. We introduced a PacI restriction enzyme site just after the open reading frame of $S$ transcriptional unit, such that the $\mathrm{S}$ transcriptional unit is flanked by MluI/PacI sites. SARS-CoV-2 S is from pCAGGS-CoV-2-S ${ }^{65}$, which codes the codon optimized $S$ from the Wuhan Hu-1 isolate (NCBI ref. seq. NC_045512.2) with a point mutation of D614G, resulting in B.1 lineage. The B.1.1.7 Spike we used carries the mutations found in GISAID Accession Number EPI_ISL 668152: del 69-70, del145, N501Y, A570D, D614G, P681H, T716I, S982A, and D1118H. The B.1.351 Spike carries the mutations D80A, D215G, del242-244, K417N, E484K, N501Y, D614G, and A701V (from EPI_ISL_745109). The Spike sequences of WT, B.1.1.7, B.1.351, and E484K are available at Genbank (Accession Numbers: MW816497, MW816498, MW816499, and MW816500; please also see Supplemental Table 2).

Sequences encoding the VSV N, P, M, G, and L proteins were also cloned into pCI vector to make expression plasmids for virus rescue, resulting in plasmids: pCI-VSV-N, pCI-VSV-P, pCI-VSV-M, pCI-VSV-G, and pCI-VSV-L. These accessory plasmids were a kind gift from Dr. Benjamin tenOever.

Generation of VSV-CoV2 spike from cDNA. In all, $4 \times 10^{5} 293 \mathrm{~T}-\mathrm{ACE} 2-$ TMPRSS2 cells per well were seeded onto collagen-I coated 6 -well plates. The next day, $2000 \mathrm{ng}$ of pEMC-VSV-EGFP-CoV2 spike, $2500 \mathrm{ng}$ of pCAGGS-T7opt ${ }^{66}, 850$ ng of pCI-VSV-N, $400 \mathrm{ng}$ of pCI-VSV-P, $100 \mathrm{ng}$ of pCI-VSV-M, $100 \mathrm{ng}$ of pCIVSV-G, $100 \mathrm{ng}$ of pCI-VSV-L were mixed with $5.5 \mu \mathrm{L}$ of Plus reagent and $8.9 \mu \mathrm{L}$ of Lipofectamine LTX (Invitrogen). Thirty minutes later, transfection mixture was applied to 293T-hACE2-TMPRSS2 cells in a dropwise fashion. Medium was replaced with DMEM-10\% FBS at 24 h post-transfection and cells maintained for 4 to 5 days until GFP positive syncytia appeared. Rescued viruses were amplified in Vero-CCL81-TMPRSS2 cells $^{41}$, then titrated on 293T-ACE2-TMPRSS2 cells and used for the virus neutralization assay.

Virus neutralization assay. In all, $5 \times 10^{4} 293$ T-hACE2-TMPRSS2 cells per well were seeded onto collagen-coated 96 -well cluster plates one day prior to use in viral neutralization assays. Virus stocks were pre-mixed with 4 -fold serially diluted serum $(1: 20$ to $1: 81,920)$ in DMEM 10\%FBS for $10 \mathrm{~min}$ at room temperature before transferring the virus plus serum mix onto target cells. Note: all sera assayed in this study were previously heat inactivated $\left(56^{\circ} \mathrm{C}\right.$ for $30 \mathrm{~min}$ ) before use in any viral neutralization studies. At $10 \mathrm{~h}$ post-infection, GFP counts were acquired by the Celigo imaging cytometer (Nexcelom Biosciences, version 4.1.3.0). Each assay was done in triplicate. For calculation of $\mathrm{IC}_{50}$, GFP counts from "no serum" conditions were set to $100 \%$; GFP counts of each condition (serum treated) were normalized to no serum control well. Inhibition curves were generated using Prism 8.4.3 (GraphPad Software) with "log (inhibitor) vs. normalized response-variable slope" settings.

Design of RBD-Fc producing Sendai virus. Sendai virus ( $\mathrm{SeV}$ ) $\mathrm{Z}$ strain cDNA sequence (AB855655.1) was generated and cloned into $\mathrm{pRS}$ vector with the addition of eGFP transcriptional unit at the head of SeV genome. The sequence of $\mathrm{F}$ transcriptional unit was from $\mathrm{SeV}$ fushimi strain (KY295909.1) due to the cloning reason. We refer to the pRS-based plasmid coding this sequence as pRS-SeVZGFP-F fushimi in this paper. For the introduction of foreign gene into $\mathrm{SeV}$, we generated additional transcriptional unit for RBD-Fc between $\mathrm{P}$ gene and $\mathrm{M}$ gene. RBD-Fc construct was generated as below; codon optimized DNA sequence of from SARS-CoV-2 spike (MN908947) in pCAGGS a gift of Dr. Florian $\mathrm{Krammer}^{65}$. S amino acids 319-541 (corresponding to the RBD domain) sequence were C-terminally fused to the Fc region of human $\operatorname{IgG}_{1}(220-449$ aa of P0DOX5.2)

Generation of recombinant Sendai virus from cDNA. In all, $2 \times 10^{5}$ BSR-T7 cells per well were seeded onto 6-well cluster plates. The next day, $4 \mu \mathrm{g}$ of pRS-SeVZGFP-Ffushimi, $4 \mu \mathrm{g}$ of pCAGGS-T7opt, $1.44 \mu \mathrm{g}$ of SeV-N, $0.77 \mathrm{ug}$ of SeV-P, $0.07 \mathrm{ug}$ of $\mathrm{SeV}$-L were mixed with $5.5 \mu \mathrm{L}$ of Plus reagent and $8.9 \mu \mathrm{L}$ of Lipofectamine LTX (Invitrogen). $30 \mathrm{~min}$ later, transfection mixtures were applied to Bsr-T7 cells in a dropwise fashion, as described previously ${ }^{66}$. At one day post transfection, medium was replaced with DMEM $+0.2 \mu \mathrm{g} / \mathrm{mL}$ of TPCK-trypsin (Millipore Sigma, \#T1426), with subsequent medium replacement each day until infection reached $100 \%$ cytopathic effect. Supernatants were stored at $-80^{\circ} \mathrm{C}$ until use in experiments.

Titration of viruses. For SeV titration, $2 \times 10^{4} \mathrm{Bsr}$-T7 cells per well were seeded onto 96 -well plates. The next day, $100 \mu \mathrm{L}$ of serially diluted virus stock (in DMEM$10 \%$ FBS) were applied to each well. GFP positive foci were counted at 24 h postinfection using a Celigo imaging cytometer (Nexcelom, Inc.). Infectivity is presented in infectious units (IU) per mL.

For VSV-CoV2 titration, $5 \times 10^{4} 293 \mathrm{~T}$-hACE2-TMPRSS2 cells per well were seeded onto a collagen-coated 96 -well plate. Serially diluted virus stocks were then 
applied to the cells, and GFP positivity was scored at $10 \mathrm{~h}$ post-infection using a Celigo imaging cytometer.

Production of proteins and purification. In all, $5 \times 10^{6} \mathrm{Bsr}-\mathrm{T} 7$ cells are seeded in $\mathrm{T} 175 \mathrm{~cm}^{2}$-flask one day before infection. Cells were infected by $\mathrm{SeV}$ at MOI of 0.1 for one hour, followed by replacement of medium with DMEM supplemented with $0.2 \mathrm{mg} / \mathrm{mL}$ TPCK-trypsin. Medium was replaced with fresh $0.2 \mathrm{mg} / \mathrm{mL}$ TPCKtrypsin containing DMEM each day until infection reached $100 \%$ cytopathic effect, at which point medium was exchanged for DMEM lacking TPCK-trypsin. Cells were incubated for additional $24 \mathrm{~h}$ to allow protein production. Supernatants were centrifuged at $400 \mathrm{xg}$ for $10 \mathrm{~min}$ at $4{ }^{\circ} \mathrm{C}$ to remove cellular debris, then passed through a $0.1 \mu \mathrm{m}$ filter (Corning $\left.{ }^{\oplus}, 431475\right)$ to remove virions, and made up to a final $0.1 \% \mathrm{TX}-100$ (Millipore Sigma, T8787) to inactivate any remaining virions. Supernatant containing RBD-Fc was applied to Protein G Sepharose (Millipore Sigma, GE17-0618-01) containing column (10 mL Polypropylene Columns; ThermoFisher, 29922), followed by 3X PBS wash and elution with $9 \mathrm{~mL}$ of $0.1 \mathrm{M}$ Glycine buffer ( $\mathrm{pH} 2.7$ ) directly into $1 \mathrm{~mL}$ of $2 \mathrm{M}$ Tris- $\mathrm{HCl}(\mathrm{pH} 10)$ neutralization buffer. Purified RBD-Fc was buffer exchanged $3 \mathrm{X}$ with PBS and concentrated in an Amicon Ultra-15 centrifugal filter (50 kDa cutoff, UFC905024). Protein concentration was determined by Bradford assay.

Serum collection. Sera were collected from Sputnik V vaccinated individuals recruited for this study by investigators at the Administración Nacional de Laboratorios e Institutos de Salud "Dr. Carlos Malbrán" (ANLIS MALBRAN) in Buenos Aires, Argentina (A.E.V, A.E, C.P.). See Human Subjects Research below. Volunteers received their first dose either on 29 December or 31 December 2020, and their second dose either on 20 January 2021 or 2 February 2021. Other patient characteristics are detailed in Table 1. Data presented in this study were from sera collected on 22 February 2021 via venipuncture directly into serum separator tubes (BD Vacutainer SST ${ }^{\mathrm{m} t}$ tubes). Serum was extracted after centrifugation as per manufacturer's instructions and was couriered the same day on dry ice to the Icahn School of Medicine at Mount Sinai (New York, USA). At least $6 \mathrm{~mL}$ of de-identified serum from each sample was received in New York and remained frozen until analysis was performed.

Human subjects research. Human subjects research was conducted following the Declaration of Helsinki and related institutional and local regulations. Studies and serum collection relating to the Sputnik vaccine at ANLIS Dr. Carlos G. Malbrán (National Administration Laboratories and Health Institutes-Carlos G. Malbrán, Argentina) were approved by the Research Ethics Committee of its Unidad Operativa Centro de Contención Biológica (UOCCB) on 9 February 2021. Written informed consent was obtained as per institutional policy.

\section{Data availability}

Source data are provided with this paper. Raw images of fluorescent microscopy data presented in Fig. 1a, c are deposited in Figshare and can be accessed using https://doi.org/ 10.6084/m9.figshare.14919732 and https://doi.org/10.6084/m9.figshare.14916627, respectively. The spike sequences of WT, B.1.1.7, B.1.351, and $\mathrm{E} 484 \mathrm{~K}$ used to generate our rcVSV-CoV- 2-S are available at Genbank (Accession Numbers: MW816497, MW816498, MW816499, and MW816500; Supplementary Table 2). Cell lines and viruses can be obtained under a materials transfer agreement from the corresponding author (benhur.lee@mssm.edu). Source data are provided with this paper.

Received: 25 May 2021; Accepted: 9 July 2021;

Published online: 26 July 2021

\section{References}

1. Huang, C. et al. Clinical features of patients infected with 2019 novel coronavirus in Wuhan, China. Lancet 395, 497-506 (2020).

2. Center for Systems Science and Engeineering at Johns Hopkins University. COVID-19 Map-Johns Hopkins Coronavirus Resource Center. https:// coronavirus.jhu.edu/map.html (2021).

3. Boni, M. F. et al. Evolutionary origins of the SARS-CoV-2 sarbecovirus lineage responsible for the COVID-19 pandemic. Nat. Microbiol. 5, 1408-1417 (2020).

4. Logunov, D. Y. et al. Safety and efficacy of an rAd26 and rAd5 vector-based heterologous prime-boost COVID-19 vaccine: an interim analysis of a randomised controlled phase 3 trial in Russia. Lancet 397, 671-681 (2021).

5. Baden, L. R. et al. Efficacy and safety of the mRNA-1273 SARS-CoV-2 vaccine. N. Engl. J. Med. 384, 403-416 (2021).

6. Polack, F. P. et al. Safety and efficacy of the BNT162b2 mRNA Covid-19 vaccine. N. Engl. J. Med. 383, 2603-2615 (2020).

7. Zhang, Y. et al. Safety, tolerability, and immunogenicity of an inactivated SARS-CoV-2 vaccine in healthy adults aged 18-59 years: a randomised, double-blind, placebo-controlled, phase 1/2 clinical trial. Lancet Infect. Dis. 21, 181-192 (2020).

8. Sadoff, J. et al. Interim results of a phase 1-2a Trial of Ad26.COV2.S Covid-19 Vaccine. N. Engl. J. Med. 384, 1824-1835 (2021).

9. Folegatti, P. M. et al. Safety and immunogenicity of the ChAdOx1 nCoV-19 vaccine against SARS-CoV-2: a preliminary report of a phase $1 / 2$, single-blind, randomised controlled trial. Lancet 396, 467-478 (2020).

10. Addetia, A. et al. Neutralizing antibodies correlate with protection from SARS-CoV-2 in humans during a fishery vessel outbreak with a high attack rate. J. Clin. Microbiol. 58, e02107-e02120 (2020).

11. Khoury, D. S. et al. Neutralizing antibody levels are highly predictive of immune protection from symptomatic SARS-CoV-2 infection. Nat. Med. https://doi.org/10.1038/s41591-021-01377-8 (2021).

12. Rambaut, A. et al COVID-19 Genomics Consortium UK (CoG-UK). Preliminary genomic characterisation of an emergent SARS-CoV-2 lineage in the UK defined by a novel set of spike mutations. virological.org https:// virological.org/t/preliminary-genomic-characterisation-of-an-emergent-sarscov-2-lineage-in-the-uk-defined-by-a-novel-set-of-spike-mutations/563 (2020).

13. Choi, B. et al. Persistence and evolution of SARS-CoV-2 in an immunocompromised Host. N. Engl. J. Med. 383, 2291-2293 (2020).

14. Davies, N. G. et al. Estimated transmissibility and impact of SARS-CoV-2 lineage B.1.1.7 in England. Science 372, eabg3055 (2021).

15. Davies, N. G. et al. Increased mortality in community-tested cases of SARSCoV-2 lineage B.1.1.7. Nature 593, 270-274 (2021).

16. Grint, D. J. et al. Case fatality risk of the SARS-CoV-2 variant of concern B.1.1.7 in England. Eur. Surveill. 26, 2100256 (2021).

17. Tegally, H. et al. Detection of a SARS-CoV-2 variant of concern in South Africa. Nature 592, 438-443 (2021).

18. Naveca, F. et al. SARS-CoV-2 reinfection by the new Variant of Concern (VOC) P. 1 in Amazonas, Brazil. virological. org. Preprint available at: https:// virological.org/t/sars-cov-2-reinfection-by-thenew-variant-of-concern-voc-p1-in-amazonas-brazil/596 (2021).

19. Dejnirattisai, W. et al. Antibody evasion by the Brazilian P.1 strain of SARSCoV-2. Cell 184, 2939-2954.e9 (2021)

20. Weisblum, Y. et al. Escape from neutralizing antibodies by SARS-CoV-2 spike protein variants. Elife 9, e61312 (2020).

21. Liu, Z. et al. Identification of SARS-CoV-2 spike mutations that attenuate monoclonal and serum antibody neutralization. Cell Host Microbe 29, 477-488.e4 (2021).

22. Greaney, A. J. et al. Comprehensive mapping of mutations in the SARS-CoV-2 receptor-binding domain that affect recognition by polyclonal human plasma antibodies. Cell Host Microbe 29, 463-476.e6 (2021).

23. Wibmer, C. K. et al. SARS-CoV-2 501Y.V2 escapes neutralization by South African COVID-19 donor plasma. Nat. Med. 27, 622-625 (2021).

24. Zhou, D. et al. Evidence of escape of SARS-CoV-2 variant B.1.351 from natural and vaccine-induced sera. Cell 184, 2348-2361.e6 (2021).

25. Wang, P. et al. Increased resistance of SARS-CoV-2 variant P. 1 to antibody neutralization. Cell Host Microbe 29, 747-751.e4 (2021).

26. Liu, Y. et al. Neutralizing activity of BNT162b2-elicited serum. N. Engl. J. Med. 384, 1466-1468 (2021).

27. Garcia-Beltran, W. F. et al. Multiple SARS-CoV-2 variants escape neutralization by vaccine-induced humoral immunity. Cell 184, 2372-2383.e9 (2021).

28. West, A. P. et al. Detection and characterization of the SARS-CoV-2 lineage B.1.526 in New York. Preprint at bioRxiv https://doi.org/10.1101/ 2021.02.14.431043 (2021).

29. Annavajhala, M. K. et al. A novel SARS-CoV-2 variant of concern, B.1.526, identified in New York. Preprint at medRxiv https://doi.org/10.1101/ 2021.02.23.21252259 (2021).

30. Voloch, C. M. et al. Genomic characterization of a novel SARS-CoV-2 lineage from Rio de Janeiro, Brazil. J. Virol. 95, e00119-e00121 (2021).

31. Sanders, R. W. \& Moore, J. P. Virus vaccines: proteins prefer prolines. Cell Host Microbe 29, 327-333 (2021).

32. Amanat, F. et al. Introduction of two prolines and removal of the polybasic cleavage site lead to higher efficacy of a recombinant spike-based SARS-CoV-2 vaccine in the mouse model. MBio 12, e02648-20 (2021).

33. Madhi, S. A. et al. Efficacy of the ChAdOx1 nCoV-19 Covid-19 vaccine against the B.1.351 variant. N. Engl. J. Med. 384, 1885-1898 (2021).

34. Logunov, D. Y. et al. Safety and immunogenicity of an rAd26 and rAd5 vector-based heterologous prime-boost COVID-19 vaccine in two formulations: two open, non-randomised phase $1 / 2$ studies from Russia. Lancet 396, 887-897 (2020).

35. Dieterle, M. E. et al. A replication-competent vesicular stomatitis virus for studies of SARS-CoV-2 spike-mediated cell entry and its inhibition. Cell Host Microbe 28, 486-496.e6 (2020).

36. $\mathrm{Li}, \mathrm{H}$. et al. Establishment of replication-competent vesicular stomatitis virusbased recombinant viruses suitable for SARS-CoV-2 entry and neutralization assays. Emerg. Microbes Infect. 9, 2269-2277 (2020). 
37. Case, J. B. et al. Replication-competent vesicular stomatitis virus vaccine vector protects against SARS-CoV-2-mediated pathogenesis in mice. Cell Host Microbe 28, 465-474.e4 (2020).

38. Baum, A. et al. Antibody cocktail to SARS-CoV-2 spike protein prevents rapid mutational escape seen with individual antibodies. Science 369, 1014-1018 (2020).

39. Yahalom-Ronen, Y. et al. A single dose of recombinant VSV- $\Delta$ G-spike vaccine provides protection against SARS-CoV-2 challenge. Nat. Commun. 11, 6402 (2020).

40. Greaney, A. J. et al. Complete mapping of mutations to the SARS-CoV-2 spike receptor-binding domain that escape antibody recognition. Cell Host Microbe 29, 44-57.e9 (2021).

41. Oguntuyo, K. Y. et al. Quantifying absolute neutralization titers against SARSCoV-2 by a standardized virus neutralization assay allows for cross-cohort comparisons of COVID-19 sera. MBio 12, e02492-20 (2021).

42. Yuan, M. et al. Structural and functional ramifications of antigenic drift in recent SARS-CoV-2 variants. Science https://doi.org/10.1126/science.abh1139. (2021).

43. Yin, R. et al. Structural and energetic profiling of SARS-CoV-2 antibody recognition and the impact of circulating variants. Preprint at bioRxiv https:// doi.org/10.1101/2021.03.21.436311 (2021).

44. Webb, N. E., Montefiori, D. C. \& Lee, B. Dose-response curve slope helps predict therapeutic potency and breadth of HIV broadly neutralizing antibodies. Nat. Commun. 6, 1-10 (2015).

45. Collier, D. A. et al. Sensitivity of SARS-CoV-2 B.1.1.7 to mRNA vaccineelicited antibodies. Nature 593, 136-141 (2021).

46. Cai, Y. et al. Structural basis for enhanced infectivity and immune evasion of SARS-CoV-2 variants. Science https://doi.org/10.1126/science.abi9745 (2021).

47. Zhu, X. et al. Cryo-electron microscopy structures of the N501Y SARS-CoV-2 spike protein in complex with ACE2 and 2 potent neutralizing antibodies. PLoS Biol. 19, e3001237 (2021).

48. Laffeber, C., de Koning, K., Kanaar, R. \& Lebbink, J. H. G. Experimental evidence for enhanced receptor binding by rapidly spreading SARS-CoV-2 variants. J. Mol. Biol. 433, 167058 (2021).

49. Finkelstein, M. T. et al. Structural analysis of neutralizing epitopes of the SARS-CoV-2 spike to guide therapy and vaccine design strategies. Viruses 13, 134 (2021)

50. Gowthaman, R. et al. CoV3D: a database of high resolution coronavirus protein structures. Nucleic Acids Res. 49, D282-D287 (2021).

51. Bos, R. et al. Ad26 vector-based COVID-19 vaccine encoding a prefusionstabilized SARS-CoV-2 Spike immunogen induces potent humoral and cellular immune responses. NPJ Vaccines 5, 91 (2020).

52. Walsh, E. E. et al. Safety and immunogenicity of two RNA-based covid-19 vaccine candidates. N. Engl. J. Med. 383, 2439-2450 (2020).

53. Corbett, K. S. et al. SARS-CoV-2 mRNA vaccine design enabled by prototype pathogen preparedness. Nature 586, 567-571 (2020).

54. Wrapp, D. et al. Cryo-EM structure of the 2019-nCoV spike in the prefusion conformation. Science 367, 1260-1263 (2020).

55. Pallesen, J. et al. Immunogenicity and structures of a rationally designed prefusion MERS-CoV spike antigen. Proc. Natl Acad. Sci. USA 114 E7348-E7357 (2017)

56. Cele, S. et al. Escape of SARS-CoV-2 501Y. V2 from neutralization by convalescent plasma. Nature 593, 142-146 (2021).

57. Hoffmann, M. et al. SARS-CoV-2 variants B.1.351 and P.1 escape from neutralizing antibodies. Cell 184, 2384-2393.e12 (2021).

58. Muik, A. et al. Neutralization of SARS-CoV-2 lineage B.1.1.7 pseudovirus by BNT162b2 vaccine-elicited human sera. Science 371, 1152-1153 (2021).

59. Shen, X. et al. SARS-CoV-2 variant B.1.1.7 is susceptible to neutralizing antibodies elicited by ancestral spike vaccines. Cell Host Microbe 29, 529-539. e3 (2021).

60. Abdool Karim, S. S. \& de Oliveira, T. New SARS-CoV-2 variants-clinical, public health, and vaccine implications. N. Engl. J. Med. 384, 1866-1868 (2021).

61. Yuan, M. et al. Structural and functional ramifications of antigenic drift in recent SARS-CoV-2 variants. Science, eabh1139-10.1126/science.abh1139 (2021)

62. Buchholz, U. J., Finke, S. \& Conzelmann, K. K. Generation of bovine respiratory syncytial virus (BRSV) from cDNA: BRSV NS2 is not essential for virus replication in tissue culture, and the human RSV leader region acts as a functional BRSV genome promoter. J. Virol. 73, 251-259 (1999).

63. Ramsburg, E. et al. A vesicular stomatitis virus recombinant expressing granulocyte-macrophage colony-stimulating factor induces enhanced T-cell responses and is highly attenuated for replication in animals. J. Virol. 79, 15043-15053 (2005).

64. Case, J. B. et al. Neutralizing antibody and soluble ACE2 inhibition of a replication-competent VSV-SARS-CoV-2 and a clinical isolate of SARS-CoV2. Cell Host Microbe 28, 475-485.e5 (2020).

65. Amanat, F. et al. A serological assay to detect SARS-CoV-2 seroconversion in humans. Nat. Med. 26, 1033-1036 (2020).
66. Beaty, S. M. et al. Efficient and robust paramyxoviridae reverse genetics systems. mSphere 2, e00376-16 (2017).

67. Elbe, S. \& Buckland-Merrett, G. Data, disease and diplomacy: GISAID's innovative contribution to global health. Glob. Chall. 1, 33-46 (2017).

68. Shu, Y. \& McCauley, J. GISAID: Global initiative on sharing all influenza datafrom vision to reality. Eur. Surveill. 22, 30494 (2017).

\section{Acknowledgements}

We gratefully acknowledge all submitting authors and collecting authors on whose work this research is based, and to all researchers, clinicians, and public health authorities who make SARS-CoV-2 sequence data available in a timely manner via the GISAID initiative ${ }^{67,68}$. We acknowledge the following finding. G.H., K.Y.O. and C.S. were supported by Viral-Host Pathogenesis Training Grant T32 AI07647 and additionally by a NRSA F31 AI154739. S.I., H.P.C., C.T.H. were supported by postdoctoral fellowships from CHOT-SG (Fukuoka University, Japan) and the Ministry of Science and Technology (MOST, Taiwan), respectively. B.L. acknowledges flexible funding support from NIH grants AI123449 and AI138921; a grant from the Department of Microbiology, Icahn School of Medicine at Mount Sinai; and the Ward-Coleman estate, which endowed the Ward-Coleman Chairs at the ISMMS. J.P.K. was supported by a COVID-19 Fast Grants award from Emergent Ventures, an initiative of the Mercatus Center at George Mason University, and by an intramural grant and other funding from the Office of the Vice Chancellor for Research at LSU Health Sciences Center Shreveport (J.P.K., M.N.A.S.). Processing costs recovered from multiple users of our standardized SARS-CoV-2 VSV pseudotyped particles provided additional support (B.L.). Work at ANLIS-MALBRAN (A.E.V., A.E., C.P.) was supported by the Ministry of Health (Ministerio de Salud), Argentina.

\section{Author contributions}

S.I., C.P., J.P.K., and B.H.L. conceived of and supervised the study. C.P., A.E.V., and A.E supervised, collected, analyzed, and provided materials relevant to this study. S.I. developed the rcVSV-CoV-2 S reverse genetics system. S.I., M.N.A.S., C-T.H., G.H., S.K., and H.-P.C. were involved in the generation of S mutant viruses. S.I, L.B., S.K., M.N.A.S., C.S.S., and K.Y.O. conducted neutralization assays. S.I. and C.-T.H. developed the Sendai virus protein expressing system and purified RBD-Fc protein. S.I. and B.H.L. analyzed the data. S.I., B.H.L., and J.P.K. wrote the paper with input from C.P. and all coauthors.

\section{Competing interests}

B.L., C.S., and K.Y.O. are named inventors on a patent filed by the Icahn School of Medicine, which includes the 293T-ACE2-TMPRSS2 (F8-2) cells used for the virus neutralization assay. J.P.K. is a consultant for BioNTech (advisory panel on coronavirus variants). The remaining authors declare no competing interests.

\section{Additional information}

Supplementary information The online version contains supplementary material available at https://doi.org/10.1038/s41467-021-24909-9.

Correspondence and requests for materials should be addressed to B.L.

Peer review information Nature Communications thanks Andreas Radbruch and the other anonymous reviewer(s) for their contribution to the peer review of this work. Peer reviewer reports are available.

Reprints and permission information is available at http://www.nature.com/reprints

Publisher's note Springer Nature remains neutral with regard to jurisdictional claims in published maps and institutional affiliations.

Open Access This article is licensed under a Creative Commons Attribution 4.0 International License, which permits use, sharing, adaptation, distribution and reproduction in any medium or format, as long as you give appropriate credit to the original author(s) and the source, provide a link to the Creative Commons license, and indicate if changes were made. The images or other third party material in this article are included in the article's Creative Commons license, unless indicated otherwise in a credit line to the material. If material is not included in the article's Creative Commons license and your intended use is not permitted by statutory regulation or exceeds the permitted use, you will need to obtain permission directly from the copyright holder. To view a copy of this license, visit http://creativecommons.org/ licenses/by/4.0/

(C) The Author(s) 2021 